\title{
Attractors of Strongly Dissipative Systems
}

by

\author{
A. G. RAMM \\ Presented by Stanisław WORONOWICZ
}

Summary. A class of infinite-dimensional dissipative dynamical systems is defined for which there exists a unique equilibrium point, and the rate of convergence to this point of the trajectories of a dynamical system from the above class is exponential. All the trajectories of the system converge to this point as $t \rightarrow+\infty$, no matter what the initial conditions are.

This class consists of strongly dissipative systems. An example of such systems is provided by passive systems in network theory (see, e.g., MR0601947 (83m:45002)).

1. Statement of the problem and assumptions. Consider the evolution problem

$$
\dot{u}=-F(u), \quad u(0)=u_{0} ; \quad \dot{u}:=\frac{d u}{d t},
$$

where $u_{0} \in D(F)$ is arbitrary, and $F: H \rightarrow H$ is a monotone and hemicontinuous operator in a Hilbert space $H$ :

$$
(F(u)-F(v), u-v) \geq 0, \quad u, v \in D(F) .
$$

Here $(u, v)$ is the inner product in $H, D(F)$, the domain of definition of $F$, is assumed to be a linear set, dense in $H$, and $F$ is assumed to be maximal monotone,

$$
R(I+F)=H,
$$

where $R(F)$ is the range of $F$. The definition of hemicontinuity can be found in [6] and we recall it in Section 2 for the convenience of the reader.

2000 Mathematics Subject Classification: 37L99, 47H05, 47H20, 47J35, 78A40, 80A30.

Key words and phrases: dissipative systems, dynamical systems, attractors, invariant manifolds, nonlinear evolution. 
Let $F$ be Fréchet differentiable, set $B:=F^{\prime}(u)$, assume that the linear operator

$$
B \geq m>0
$$

is selfadjoint, and define $A:=B^{-1}$. The operator $A$ is selfadjoint and bounded, $\|A\| \leq m^{-1}$. One may replace the assumption of the Fréchet differentiability by the assumptions of strong monotonicity

$$
(F(u)-F(v), u-v) \geq m\|u-v\|^{2}
$$

plus hemicontinuity, but this is not important for our purposes. The Fréchet differentiability implies hemicontinuity.

The above standing assumptions are not repeated below.

The system described by equation (1) is called dissipative if assumption (2) holds, and strongly dissipative if assumptions (2) and (4) hold.

Such systems arise in many applications in physics, where the energy is dissipated. For example, the theory of passive networks developed in $[2$, Chapter 3] and [3] is based on the equation (1). In [5] one finds a justification of a method for calculating slow invariant manifolds for dissipative systems. In [7] one finds many examples and a general discussion of dissipative dynamical systems.

We are interested in finding forward invariant manifolds (sets) for systems described by equation (1) such that these manifolds are minimal global attractors, which consist of just one point, the unique equilibrium point for (1).

It will be shown that for any initial data $u_{0}$ the trajectories $u(t):=$ $u\left(t ; u_{0}\right)$ of the system (1) as $t \rightarrow+\infty$ converge at an exponential rate to this point, so that $F\left(u\left(\infty ; u_{0}\right)\right)=0$ for any $u_{0}$.

A proper subset $M \subset H$ is called a forward (or positively) invariant set for (1) if $u_{0} \in M$ implies $u(t) \in M$ for all $t>0$.

A forward invariant set $\mathcal{A}$ is called a global attractor for (1) if

$$
\lim _{t \rightarrow \infty} d(u(t), \mathcal{A})=0,
$$

where $d(u, \mathcal{A})$ is the distance from $u$ to $\mathcal{A}, u(t)=u\left(t ; u_{0}\right)$ is the solution to (1), and (5) holds for every $u_{0} \in H$.

A subset of the set $\mathcal{A}$ may also be a global attractor. Then one may be interested in finding a minimal global attractor.

In Theorem 1 of Section 2 such a minimal global attractor consists of just one point. This point is the unique equilibrium point for the system (1) under the assumptions of Theorem 1.

A forward invariant set may often be a manifold. In this case it is called a forward invariant manifold for the system (1). 
A solution to (1) is called global if it is defined for all $t>0$. Under the assumptions of Theorem 1, as we prove in Section 2, equation (1) has a unique global solution for every initial data $u_{0} \in H$, the limit $u(\infty):=\lim _{t \rightarrow \infty} u(t)$ exists, and $F(u(\infty))=0$. Such results are basic in the monograph [4], where they form a basis of the Dynamical Systems Method (DSM) for solving the stationary equation $F(u)=0$. The ideas from [4] are used in this paper.

Our main result, Theorem 1 in Section 2, says that for strongly dissipative dynamical systems satisfying the assumptions of that theorem, a minimal global attractor exists, consists of just one point, this point is the equilibrium point for the system (1) (so it is an invariant set for the system (1)), and all the trajectories of the system (1), that is, solutions $u=u\left(t ; u_{0}\right)$ to problem (1) for arbitrary initial data $u_{0}$, converge at an exponential rate to that point as $t \rightarrow \infty$.

This is a typical situation for strongly dissipative dynamical systems.

The main result is illustrated by an example in Section 3. The presentation in our short note is essentially self-contained.

The steps of our proof include: (a) proof of the uniqueness of the solution, (b) proof of the existence of a global solution, (c) and (e) derivation of a priori bounds on the solution, (d) proof of the existence of $u(\infty)$, (e) derivation of the estimate (13) and proof that $u(\infty)$ solves the equation $F(u(\infty))=0$, (f) proof that the trajectory remains in a ball of a fixed radius for all times.

The example we consider is the Ginzburg-Landau boundary-value problem, but other examples can be provided easily (see [2, Chapter 3]).

2. Results. Our main result is:

TheOREM 1. Under the assumptions of Section 1 problem (1) has a unique global solution $u(t)$, the limit $u(\infty)$ exists, $F(u(\infty))=0$, and we have $\|u(t)-u(\infty)\| \leq c e^{-m t}$, where $m>0$ is the constant from (4).

Proof. (a) Problem (1) has at most one solution: if $u$ and $v$ solve (1), then $w:=u-v$ solves the problem

$$
\dot{w}=-[F(u)-F(v)], \quad w(0)=0 .
$$

Multiply (6) by $w$ and use (2) to get

$$
(\dot{w}, w) \leq 0, \quad w(0)=0 .
$$

This implies $w=0$, so $u=v$.

(b) Problem (1) has a unique global solution because $F$ is maximal monotone (see, e.g., [6, p. 174, Komura-Kato Theorem]; the Fréchet differentiability is not used in the proof of this result). This solution is an absolutely continuous function $[0, \infty) \rightarrow H$ and $\left\|u^{\prime}\right\|_{L^{\infty}(0, \infty ; H)} \leq c\left(u_{0}\right)$ 
([6, p. 174]). Moreover, $u(t) \in D(F)$ for all $t>0$. Therefore the expression $F^{\prime}(u(t)) \dot{u}(t)=-B F(u(t))$ makes sense on the trajectory $u(t)$, and $F(u(t)) \in D(B)$.

If $F$ is a locally Lipschitz monotone operator, then the proof of the global existence of the solution to problem (1) is much shorter than the proof of the Komura-Kato Theorem and can be found in [4] and [6]. The idea of the proof of the Komura-Kato Theorem is to approximate $F$ by a Lipschitz operator using Yosida approximation, and then pass to the limit as the parameter of the approximation tends to zero. The Yosida approximation is defined by $F_{a}:=a^{-1}\left(I-J_{a}\right)$, where $J_{a}:=(I+a F)^{-1}, a=$ const $>0$. The operator $F_{a}$ is maximal monotone and Lipschitz with Lipschitz constant $a^{-1}$. For each $v \in D(F)$ the sequence $\left\|F_{a}(v)\right\|$ is increasing and bounded, hence it converges. If $F$ is maximal monotone, condition (4) holds, and $u \in D(F)$,

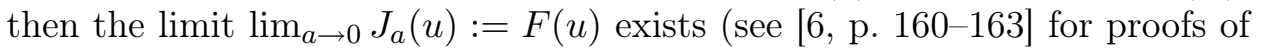
these statements).

(c) This solution is bounded on every interval:

$$
\|u(t)\| \leq c t .
$$

By $c>0$ we denote various constants independent of $t$.

Let us derive (8). Multiply (1) by $u$, let $g:=g(t):=\|u(t)\|$, and get

$$
\begin{aligned}
g \dot{g} & =(\dot{u}, u)=-(F(u)-F(0), u)-(F(0), u) \\
& \leq-(F(0), u) \leq c g, \quad c:=\|F(0)\| .
\end{aligned}
$$

Inequality (9) implies (8). Thus, the global solution to (1) is bounded on every fixed time interval. Actually, the solution $u(t)$ to (1) is uniformly bounded on $[0, \infty)$, as follows from (e) below.

(d) Let us now prove the existence of $u(\infty)$ and estimate the rate of convergence of $u(t)$ to $u(\infty)$.

Let $h(t)=\|F(u(t))\|$. Using (1) and (4) one gets

$$
h \dot{h}=-(B F(u(t)), F(u(t))) \leq-m h^{2} .
$$

Here we have used the inclusion $F(u(t)) \in D(B)$ and the inequality (4).

Since $h \geq 0$, inequality (10) implies

$$
h(t) \leq c e^{-m t}, \quad c=h(0) .
$$

From (1) and (11) it follows that

$$
\|\dot{u}(t)\| \leq c e^{-m t} .
$$

From (12) and the Cauchy criterion the existence of the limit $u(\infty)$ follows. The limit $u(\infty)$ also exists if a weaker estimate $\|\dot{u}(t)\| \in L^{1}\left(\mathbb{R}_{+}\right)$is satisfied, where $\mathbb{R}_{+}=[0, \infty)$. 
(e) To estimate the rate of convergence of $u(t)$ to $u(\infty)$, let us integrate (12) from $t$ to $\infty$ :

$$
\|u(t)-u(\infty)\| \leq \int_{t}^{\infty} c e^{-m s} d s \leq c m^{-1} e^{-m t} .
$$

From (1), (12), and the demicontinuity of $F$ it follows that $F(u(\infty))=0$.

Let us give a detailed explanation of this statement. Denote by $\rightarrow$ weak convergence in $H$, by $\rightarrow$ strong convergence in $H$, and recall that hemicontinuity means that $F(u+s v) \rightarrow F(u)$ as $s \rightarrow+0$, where $s \in \mathbb{R}_{+}$, $u, v \in D(F)$ and $u+s v \in D(F)$, while demicontinuity means that $u \rightarrow w$ implies $F(u) \rightarrow F(w)$, where $u, w \in D(F)$. It is known ([1, p. 98]) that a monotone hemicontinuous operator is demicontinuous on sets on which $F$ is bounded. In our case, the trajectory $u(t)$ is such a set.

Since in [1, p. 98], the demicontinuity of a monotone hemicontinuous operator at a point $v$ is derived under an additional assumption that $v$ is an interior point of the domain $D(F)$, and since under our assumptions $F$ is densely defined and $D(F)$ may have no interior points, let us prove that $F(u) \rightarrow 0$ and $u \rightarrow v$ imply $F(v)=0$, provided that $F$ is monotone and hemicontinuous. By the monotonicity of $F$ one has $(F(u)-F(v-s h), u-v+s h) \geq 0$ for any $h \in D(F)$ and any $s>0$. Passing to the limit $F(u) \rightarrow 0$ and $u \rightarrow v$, one gets $(-F(v-s h), h) \geq 0$. Using the hemicontinuity of $F$ and letting $s \rightarrow+0$ yields $(-F(v), h) \geq 0$ for all $h \in D(F)$. Since $D(F)$ is dense in $H$ by assumption, one concludes that $F(v)=0$. In our case $v:=u(\infty)$.

From (12) one gets $\lim _{t \rightarrow \infty}\|\dot{u}(t)\|=0$. Together with (1) this implies $F(u(t)) \rightarrow 0$ as $t \rightarrow \infty$. Inequality (13) implies $\lim _{t \rightarrow \infty}\|u(t)-u(\infty)\|=0$. This, as we have proved, yields $F(u(\infty))=0$. The desired relation is proved.

(f) One can estimate the radius $R$ of the ball $B\left(u_{0}, R\right):=\left\{u:\left\|u-u_{0}\right\|\right.$ $\leq R\}$ to which the orbit of the solution $u(t)$ belongs: integrate (12) from 0 to $t$ to get

$$
\left\|u(t)-u_{0}\right\| \leq c m^{-1} \quad \forall t>0 .
$$

Therefore, the solution $u(t)$ stays in the ball $B\left(u_{0}, h(0) m^{-1}\right)$ for all $t>0$.

The proof of Theorem 1 is complete.

Remark 2.1. Let $N:=\{u: F(u)=0\}$. Theorem 1 implies that $N \subset \mathcal{A}$. Indeed, the set $N$ consists of equilibrium points for the system (1). The set $N$ is an invariant set for the system (1). However, if $\operatorname{dim} N>1$, then the limit $u\left(t ; u_{0}\right)$ of the solution to (1) as $t \rightarrow \infty$ depends on the choice of $u_{0}$. Therefore, if $\operatorname{dim} N>1$, then the global attractor for the system (1) is no longer just one point, while under the assumptions of Theorem 1 the set $N$ consists of one element, and the global attractor is just one point. If the 
operator $F$ is monotone but the assumption (4) is dropped, then the set $N$ may have arbitrarily many elements.

3. Example. Consider the Ginzburg-Landau (GL) evolution problem

$$
\dot{u}=\Delta u-u^{3}+f \quad \text { in } D \times[0, \infty), \quad u(0)=u_{0},\left.\quad u\right|_{S}=0,
$$

where $D \subset \mathbb{R}^{3}$ is a bounded domain with a sufficiently smooth boundary $S$, $H=L^{2}(D), f \in H, F(u)=-\Delta u+u^{3}-f, D(F)=H^{2} \cap H_{0}^{1}, H^{l}=H^{l}(D)$ is the Sobolev space, $H_{0}^{1}$ is the closure of $C_{0}^{\infty}(D)$ in the norm of $H^{1}, u_{0} \in H$. One has

$$
F^{\prime}(u) v=B v=-\Delta v+3 u^{2} v, \quad B \geq m>0,
$$

where $m>\Lambda>0$, and $\Lambda>0$ is the first eigenvalue of the Dirichlet Laplacian in $D$.

The set $N$ is the set of solutions to the problem

$$
-\Delta u+u^{3}-f=0 \quad \text { in } D,\left.\quad u\right|_{S}=0 .
$$

Problem (15) has a unique solution: if $u$ and $v$ solve (15) then $w:=u-v$ solves the problem

$$
-\Delta w+u^{3}-v^{3}=0 \quad \text { in } D,\left.\quad u\right|_{S}=\left.v\right|_{S}=0 .
$$

Multiply (16) by $w$, integrate over $D$ and then by parts, to get

$$
\int_{D}\left[|\nabla w|^{2}+\left(u^{3}-v^{3}\right)(u-v)\right] d x=0,\left.\quad u\right|_{S}=\left.v\right|_{S}=0 .
$$

This implies that $w=0$, so $u=v$. Uniqueness of the solution to (15) is proved.

To prove the existence of the solution to (15), one uses the known result: coercive monotone operators are surjective (see, e.g., [4]). The coercivity of the map $F, F(u)=-\Delta u+u^{3}-f$, can be easily checked.

Thus, the global attractor for problem (14) with the given $f$ consists of one point, the solution to (15). This point is an invariant manifold for the problem (14).

In other applied problems the dimension of $N$ may be greater than one. In that case $\mathcal{A}$ may have more than one point.

\section{References}

[1] K. Deimling, Nonlinear Functional Analysis, Springer, Berlin, 1985.

[2] A. G. Ramm, Theory and Applications of Some New Classes of Integral Equations, Springer, New York, 1980.

[3] —, Stationary regimes in passive nonlinear networks, in: Nonlinear Electromagnetics, P. Uslenghi (ed.), Academic Press, New York, 1980, 263-302.

[4] -, Dynamical Systems Method for Solving Operator Equations, Elsevier, Amsterdam, 2007. 
[5] A. G. Ramm, Slow invariant manifolds for dissipative systems, J. Math. Phys. 50 (2009), 042701.

[6] R. Showalter, Monotone Operators in Banach Space and Nonlinear Partial Differential Equations, Amer. Math. Soc., Providence, RI, 1997.

[7] R. Temam, Infinite-Dimensional Dynamical Systems in Mechanics and Physics, Springer, New York, 1997.

A. G. Ramm

Mathematics Department

Kansas State University

Manhattan, KS 66506-2602, U.S.A.

E-mail: ramm@math.ksu.edu

Received February 22, 2009;

received in final form April 8, 2009 